ware program that does not round off at 2 decimal places. Thus, in both random- and fixed-effects modelling, the confidence intervals approached but did not incorporate $\mathrm{I}$. The summary effect estimate for the comparison in Fig. IA ( $\beta$-blocker v. placebo in trials enrolling younger patients) with the random-effects model is 0.862 (95\% CI $0.746-0.996 ; p=$ 0.044 ); This result (for which the 95\% CI in our original publication was truncated, rather than rounded, to $0.74^{-0.99)}$ clearly supports the interpretation stated in our paper that $\beta$-blockers are more efficacious than placebo in younger patients. Similarly, the summary effect estimate for the comparison in Fig. 2B ( $\beta$-blocker $v$. other antihypertensives in trials enrolling older patients) with the random-effects model is 1.066 (95\% CI I.00I-I.I35, incorrectly reported in our original publication as 1.06 with 95\% CI I.OI-I.IO; $p=0.047$ ); this result supports our interpretation that $\beta$-blockers were less efficacious than other antihypertensive agents in older patients. Unfortunately, this estimate and $95 \%$ CI were incorrectly reported in our paper; in rounded form, these values should have been reported as I.07 (95\% CI I.OI-I.IO). ${ }^{1}$

Andrea Siebenhofer and colleagues note that our definition of the composite outcome was unclear. The initial version of our meta-analysis was substantially longer than the final published version and thus contained far more detail on trial entry criteria, baseline characteristics and outcome definitions. For publication purposes, we were asked to shorten the manuscript, and we regret that in doing so we inadvertently caused confusion for some readers. To clarify, our primary analyses were for the composite outcome of cardiovascular deaths (or all-cause deaths where cardiovascular deaths were not reported), MI or stroke. In light of the concerns of Siebenhofer and colleagues about the $\mathrm{I} 2$ trials included in Figs. $2 \mathrm{~A}$ and $2 \mathrm{~B}$ of our meta-analysis, it is important to point out that the composite outcomes incorporated cardiovascular mortality in 9 of those trials, total mortality in 2 and cardiac mortality in $\mathrm{I}$. Ten of the $\mathrm{I} 2$ trials incorporated fatal and nonfatal MI and stroke in their composite outcomes (one trial included only fatal and nonfatal MI and another included only fatal MI and stroke). We did not have access to the primary study data and therefore relied on the end-point definitions and methods of classification used by the primary trialists (recognizing that this was consistent within trials and thus would be identical across treatment groups within each trial).

In their table, Siebenhofer and colleagues report a different number of events in the composite outcome for the CAPPP trial than we did. Although we defined the composite event rates as the proportion of patients experiencing any of the composite events (and thus counted each participant only once), Sienbenhofer and colleagues appear to have pooled the number of events for the individual end points separately to arrive at their composite event rates. However, this individual counting of events assumes that they are independent and that no patients suffered more than one event; this is clearly not the case and we therefore favour our approach (which was that taken by the CAPPP authors in their primary publication $^{2}$ ).

Siebenhofer and colleagues and Bo Carlberg questioned our inclusion of data from CAPPP and STOP2 (mixed $\beta$ blocker studies). We discussed the reasons for including these trials in our paper, ${ }^{1}$ where we also reported 2 sensitivity analyses that address these concerns.

Finally, Carlberg points out that the meta-analysis that he and his colleagues conducted differed from ours in that they examined the effect of $\beta$ blocker treatment on the incidence of MI, stroke or death separately and based their conclusions on the excess risk of stroke observed in patients treated with $\beta$-blockers. On the other hand, we focused on the composite end point of all 3 conditions together to account for competing risks and the potential for survivor bias. As discussed in our paper, an agent with a beneficial effect on one end point may appear to have a detrimental effect on another end point, given that treated patients survive without the first end point for long enough that the second end point occurs.

\section{Nadia Khan}

Division of General Internal Medicine University of British Columbia

Vancouver, BC

\section{Finlay McAlister}

Division of General Internal Medicine University of Alberta

Edmonton, Alta.

\section{REFERENCES}

I. Khan N, McAlister FA. Re-examining the efficacy of $\beta$-blockers for the treatment of hypertension: a meta-analysis. CMAJ 2006;174(12):1737-42.

2. Hansson L, Lindholm LH, Niskanen L, et al. Effect of angiotensin-converting-enzyme inhibition compared with conventional therapy on cardiovascular morbidity and mortality in hypertension: the Captopril Prevention Project (CAPPP) randomised trial. Lancet I999;353:6II-6.

DOI:I0.I503/cmaj.I060200

\section{Coombs' testing}

\section{and neonatal}

\section{hyperbilirubinemia}

Although we fully agree with Michael Sgro and colleagues regarding the need for early identification and effective management of neonatal hyperbilirubinemia, we were surprised to see their recommendation that the Coombs' test be used to screen for hyperbilirubinemia in all infants born to mothers with type O blood. ${ }^{1}$ Although the Coombs' or direct antibody test (DAT) is an important test when trying to identify the cause of neonatal hyperbilirubinemia, recent studies have shown that it has extremely limited usefulness in predicting the development of significant hyperbilirubinemia.

The DAT has been shown to have a positive predictive value of $12 \%-53 \%$ and a sensitivity of $15 \%-64 \%$ for the subsequent development of hyperbilirubinemia, which limits its usefulness as a screening test (Table I). ${ }^{2-4}$ Herschel and colleagues found that in comparison with measurements of end-tidal carbon monoxide concentration, the DAT showed only $8.5 \%$ sensi- 
tivity, $97.6 \%$ specificity and a positive predictive value of $25 \%$ for the detection of hemolysis in neonates. ${ }^{3}$

In addition to having very low predictive ability, the DAT is costly when used as a screening test. US studies of the costs of evaluating neonatal jaundice have reported the cost per test to be US\$17-\$47. . $^{2,5,6}$

Newman and colleagues concluded that the investigation of hyperbilirubinemia should be individualized, with more aggressive investigation of infants with early onset or severe hyperbilirubinemia. ${ }^{6}$ Holtzman has also stressed the need for critical appraisal of strategies intended to identify infants with hyperbilirubinemia.?

In Calgary, routine DAT testing is being phased out in favour of a comprehensive hospital- and community-based transcutaneous bilirubinometry program. We believe that it provides a convenient, rapid, painless, cost-effective and accurate screening assessment for hyperbilirubinemia in the term and near-term neonate, particularly when incorporated into routine well-baby visits by public health nurses. ${ }^{8}$

We believe that the DAT should be reserved for diagnostic purposes in children with early or clinically significant hyperbilirubinemia.

\section{Stephen Wainer}

Assistant Clinical Professor

Jack Rabi

Assistant Professor

Department of Pediatrics

University of Calgary

Martha Lyon

Section Head

Pediatric \& Neonatal Clinical

Biochemistry

Calgary Laboratory Services

Calgary, Alta.

\section{REFERENCES}

I. Sgro M, Campbell D, Shah V. Incidence and causes of severe hyperbilirubinemia in Canada. CMAJ 2006;175(6):587-90.

2. Meberg A, Johansen KB. Screening for neonatal hyperbilirubinemia and $\mathrm{ABO}$ alloimunization at the time of testing for phenylketonuria and congenital hypothyreosis. Acta Paediatr 1998;87:1269-74.

3. Herschel M, Karrison T, Wen M, et al. Evaluation of the direct antiglobulin (Coombs') test for identifying newborns at risk for hemolysis as determined by end-tidal carbon monoxide concentration (ETCOc); and comparison of the Coombs' Test with ETCOc for detecting significant jaundice. J Perinatol 2002;22:34I-7.

4. Dinesh D. Review of positive direct antiglobulin tests found on cord blood sampling. J Paediatr Child Health 2005;4I(9-I0):504-7.

5. Suresh GK, Clark RE. Cost-effectiveness of strategies that are intended to prevent kernicterus in newborn infants. Pediatrics 2004;II4:917-24.

6. Newman TB, Easterling MJ, Goldman ES. Laboratory evaluation of jaundice in newborns: frequency, cost and yield. Am J Dis Child I990;I44:364-8.

7. Holtzman NA. Management of hyperbilirubinemia: quality of evidence and cost. Pediatrics 2004; II4:I086-8.

8. Engle WD, Jackson GL, Stehel EK, et al. Evaluation of a transcutaneous jaundice meter following hospital discharge in term and near-term neonates. $J$ Perinatol 2005;25:486-9o.

DOI:I0.I503/cmaj.I0602I4

\section{[The authors respond:]}

We thank Stephen Wainer and colleagues for their comments on our recent article. ${ }^{1}$ We agree that using any test in isolation, including the Coombs' test, is not the most effective way to identify infants at risk of neonatal hyperbilirubinemia. Our recommendation for Coombs' testing was not for all infants whose mothers had type O+ blood, only for those who had risk factors for hyperbilirubinemia or were already jaundiced at the time of discharge.

Despite existing guidelines from the American Academy of Pediatrics ${ }^{2}$ and the Canadian Paediatric Society ${ }^{3}$ recommending identification of newborns at risk and close follow-up of these infants, our data clearly demonstrate that

Table 1: Use of the direct antibody test to predict the development of hyperbilirubinemia in newborns

\begin{tabular}{lcccc}
\hline Study & PPV (\%) & NPV (\%) & Sensitivity & Specificity \\
\hline Meberg and Johansen $^{2}$ & 12 & 96 & 64 & 65 \\
Herschel et al $^{3 *}$ & 53 & 89 & 15 & 98 \\
Dinesh $^{4} \dagger$ & 23 & 92 & 15 & 95 \\
\hline
\end{tabular}

Note: $\mathrm{PPV}=$ positive predictive value, NPV = negative predictive value.

*Results for infants born to nonsmoking mothers.

†Calculated results from data based on need for phototherapy.

severe neonatal hyperbilirubinemia continues to occur at an alarming rate in Canada. The most common cause in our population was $\mathrm{ABO}$ incompatibility; this needs to be emphasized to pediatricians and primary health care practitioners.

Many strategies have been postulated as being cost-effective in preventing severe neonatal hyperbilirubinemia. We welcome the use of strategies coupling clinical suspicion of risk of hyperbilirubinemia at the time of discharge with close outpatient monitoring. Transcutaneous bilirubinometers, although very useful within a clinical context, may not always serve as a substitute for a serum bilirubin measurement when the bilirubin concentration reaches levels at which phototherapy is required. ${ }^{4,5}$ No reported strategies using transcutaneous bilirubinometers have yet been proven to be cost-effective, ${ }^{6}$ largely because the prevalence of long-term neurological sequelae of severe hyperbilirubinemia is not yet known.

\section{Michael Sgro \\ Douglas Campbell \\ Department of Pediatrics \\ St. Michael's Hospital \\ Vibhuti Shah \\ Department of Pediatrics \\ Mount Sinai Hospital \\ Toronto, Ont.}

\section{REFERENCES}

I. Sgro M, Campbell D, Shah V. Incidence and causes of severe hyperbilirubinemia in Canada. CMAJ 2006;175(6):587-90.

2. American Academy of Pediatrics Subcommittee on Hyperbilirubinemia. Management of hyperbilirubinemia in the newborn infant 35 or more weeks of gestation. Pediatrics 2004;II4:297-316.

3. Fetus and Newborn Committee, Canadian Paediatric Society. Approach to the management of hyperbilirubinemia in term newborn infants. Paediatr Child Health I999;4(2):I6I-4.

4. Engle WD, Jackson GL, Stehel EK, et al. Evaluation of a transcutaneous jaundice meter following hospital discharge in term and near-term neonates. Perinatol 2005;25:486-9o.

5. Danayan KC, Sgro M, McGovern V, et al. Transcutaneous bilirubin measurement in jaundiced newborns. Paediatr Child Health 2006; II(Suppl B):26B.

6. Suresh GK, Clark RE. Cost-effectiveness of strategies that are intended to prevent kernicterus in newborn infants. Pediatrics 2004;114:917-24.

DOI:I0.I503/cmaj.Io60244

In their commentary ${ }^{1}$ on our recent article, ${ }^{2}$ Jeffrey Maisels and Thomas 